\title{
Improving Patient-Pharmacist Encounters with Over-The-Counter Medications: A Mixed-Methods Pilot Study
}

\author{
Aaron M. Gilson, MS MSSW, PhD ${ }^{1}$; Ka Z Xiong, $\mathrm{MPH}^{2}$; Jamie A. Stone, $\mathrm{MS}^{3}$; Nora Jacobson, PhD ${ }^{4}$; Cynthia Phelan, PhD, $R N^{5}$; \\ Apoorva Reddy, BS (Graduate Student) ${ }^{3}$; Michelle A. Chui, PharmD, PhD ${ }^{3}$ \\ ${ }^{1}$ Sonderegger Research Center, University of Wisconsin-Madison School of Pharmacy \\ ${ }^{2}$ Social and Administrative Sciences Division, University of Wisconsin-Madison School of Pharmacy \\ ${ }^{3}$ Social and Administrative Sciences Division, University of Wisconsin-Madison School of Pharmacy \\ ${ }^{4}$ Institute for Clinical and Translational Research, Community Academic Partnerships Program, University of Wisconsin-Madison \\ ${ }^{5}$ Center for Nursing Research and Practice, Aurora Sinai Hospital
}

\begin{abstract}
Background and Objectives: Over-the-counter (OTC) medication use has increased safety risks for adults older than 65 . Most older adults purchase OTC medications from community pharmacies, where the considerable distance or visual obstructions between the prescription area and OTC aisles undermine pharmacists' ability to assist patients with OTC medication decisions. An innovative redesign of an abbreviated medication section specifically for older adults (called the Senior Section ${ }^{T M}$ ) can facilitate pharmacy staff/patient interaction, potentially improving safe medication selection and use. This study evaluated the impact of the Senior Section on the frequency and content of OTC encounters between pharmacy staff and patients.

Research Design and Methods: An intervention mixed-methods design generated data from patient OTC encounters, and interviews with two pharmacists and two technicians, throughout the study. NVivo was used to code interview transcripts, and frequencies and chi-square analyses demonstrated pre/post-intervention comparisons for the OTC encounter variables.

Results: After Senior Section implementation, pharmacy staff were more likely to initiate (and be involved in) patient encounters, address more topics or problem/symptoms, provide details about OTC products, discuss appropriateness of OTC use, and discuss medication classes highlighted in the Senior Section. Pharmacy staff were less likely to need to leave the prescription department for extended periods; they also had fewer prolonged encounters or encounters about product location. Importantly, the Senior Section did not impede pharmacy workflow.

Discussion and Implications: The Senior Section prompted more frequent, effective, and efficient engagements between pharmacy staff and patients, which may substantially reduce OTC-related harms among older adults.
\end{abstract}

Keywords: Medication Safety, System Redesign, Pharmacist-Patient Communication

\section{Background}

Over-the-counter (OTC) medication use in older adults (age $65+)^{1}$ has long represented a critical but often-neglected health safety issue. ${ }^{2}$ Indeed, an estimated 1.2 million older adults are at major risk of drug-drug interactions due to inappropriate concurrent use of two or more OTC medications. ${ }^{3}$ The potential for OTC-related harms has long been acknowledged in the healthcare field, precipitating development of consensus-based or evidence-based clinical practice resources to reduce or avoid medication misuse in older adults. ${ }^{4,5}$ Despite such patient care resources, older adults' involvement in emergency hospitalizations for adverse drug events increases annually, including for OTC medications. ${ }^{6}$ Even when

Corresponding author: Michelle A. Chui, PharmD, PhD Director, Sonderegger Research Center

Associate Professor, Social and Administrative Sciences Div. University of Wisconsin-Madison School of Pharmacy 777 Highland Avenue, Madison, WI 53705

Email: michelle.chui@wisc.edu practitioners are aware of potential dangers inherent in many OTC products, their knowledge cannot improve patient care unless they engage around these issues when older adults are seeking to address their healthcare needs. Working collaboratively with patients is essential for improving medication safety.

Community retail pharmacies offer a valuable venue for improving OTC medication safety and reducing adverse events (see, for example, ${ }^{7-9}$ ). Not only are community pharmacies where most older adults purchase OTC medications, but they also allow for access to a pharmacist who can provide free and authoritative medication guidance to patients when choosing OTC products - pharmacists are trained to assess whether an OTC medication is safe to use based on patients' physical symptoms and chronic health conditions. Unfortunately, studies document numerous barriers to pharmacist-patient OTC consultations, especially erroneous patient perceptions that OTC medications are safe, leading to patient beliefs that expert advice from healthcare professionals is not required when selecting these products for use. ${ }^{10}$ It is ironic, therefore, that prior community pharmacy interventions related to OTC 
products have typically focused on enhancing effective communication during OTC consultations, even though patients' reluctance to initiate engagement with pharmacists continues to contribute to consultation barriers. ${ }^{11}$

The interior design and structural layout common to most community pharmacies (and the mass-merchandise stores in which pharmacies are frequently located), and physical barriers (e.g., signage, walls, and corners), can impede visual observation of the patient. When OTC encounters occur, they likely consume more time due to the need for pharmacy staff to travel across this distance to where the product is located. Indeed, a 2015 pharmacists survey found that OTC encounters were most likely to occur in pharmacy product aisles, and pharmacists identified the "location of nonprescription and self-care products readily accessible by pharmacists" (p. 6) as the most important factor in promoting frequent patient engagement. ${ }^{12}$ In addition, the time pressure from dispensing responsibilities may reduce pharmacists' willingness to leave the prescription area. Addressing these environmental issues may enhance pharmacy staff ability to guide patients in their OTC medication selection and use.

\section{The Senior Section ${ }^{T M}$}

This project implemented an innovative OTC intervention, while partnering with an advisory group of older adults and stakeholders from a mass merchandise retail organization, to correct a principal system design obstacle to OTC encounters and consequently promote safer OTC use. ${ }^{13}$ Beginning in 2016, a redesign of the physical layout of the OTC aisles (the Senior Section) was implemented in four Midwest pharmacies to facilitate effective engagement between pharmacy staff and community-dwelling older adults; a recent article describes the participatory design process leading to the Senior Section arrangement. ${ }^{14}$

The Senior Section is a specially-curated section, representing four medication classes: Pain, sleep, cough/cold, allergy. Select medications including, for example, diphenhydramine products, aspirin (when used for pain), and cough/cold combination products were excluded from this section because they are high-risk for older adults. ${ }^{5}$ The Senior Section was proximal to the prescription area and within a direct sight line to pharmacists and technicians. In addition, cautionary signage was displayed in the main aisle to identify OTC medication categories (e.g. sleep, cough/cold, pain) that generally have higher risks for older adults, and encouraged older adults to visit the Senior Section and ask the pharmacist. These features were meant to heighten older adult awareness of high-risk OTC medications and facilitate communication with pharmacists, who can help inform decisions, while offering an intervention that reduces pharmacy staff barriers and facilitates OTC-related patient encounters. However, the Senior Section could unintentionally increase pharmacy staff workload through significantly more OTC consultations or interrupt other pharmacy duties.

\section{Objectives}

This investigation identified the Senior Section's impact on the frequency and content of pharmacy staff/patient OTC consultations. Information about the characteristics, quantity, and type of pharmacy staff/patient OTC encounters, as well as comparing encounters across pre- and post-implementation of the Senior Section, were used to assess intervention feasibility, preliminary effectiveness, and sustainability. A hypothesis underlying the study intervention was that, after introducing the Senior Section, OTC encounters would become more frequent due to the new section's proximity to the pharmacy. OTC encounter time was expected to decrease because pharmacists/technicians would not need to leave the prescription department to assist patients. As a result, the Senior Section was expected to minimally affect pharmacy staff workload.

\section{Research Design and Methods}

An intervention mixed-methods design ${ }^{15}$ was used to explore the Senior Section's OTC safety intervention effects. The multiple methods comprising this approach involved pre- and post-assessment of the intervention and included both quantitative and qualitative elements.

\section{Intervention}

The conceptual framework and process used to design the Senior Section was based on the human factors framework called System Engineering Initiative for Patient Safety (SEIPS). ${ }^{16}$ The Senior Section was designed to achieve the dual objective of improving medication safety and improving efficiency of pharmacy staff/patient encounters, defined to involve the frequency, duration, and focus of the encounter, and has been described in previous publications. ${ }^{13,14}$

\section{Recruitment, Participants, \& Setting}

Recruitment meetings were scheduled between the pharmacy manager and project $\mathrm{PI}$ at times convenient for pharmacy staff. The research team introduced the study and interested participants either signed up then or took an information sheet. From a single pharmacy site of a mass merchandise store in Wisconsin, the following pharmacy staff were recruited:

- the two full-time pharmacists who worked at that store, and

- two technicians (from an unknown but larger number of technicians) who worked at least 20 hours/week and who expressed interest in participating in the study.

The pharmacy is located in a Wisconsin community with a population of about 37,000 people, representing a diverse age, gender, and race demographic.

http://suburbanstats.org/population/wisconsin 
This was the sole pharmacy site for which it was appropriate to conduct a pre/post mixed-methods analysis, because of an unexpected event - the closure of the mass merchandise store chain. Post-implementation data collection (see OTC Encounter Form section below) had already concluded at this particular pharmacy by the time the store closure plan was announced. Data collection for all other pharmacies occurred during a period in which pharmacy staff were aware of the impending store closures, which would likely have affected the thoroughness and reliability of the information being compiled. As a result, the information presented in this article represents the only data that were not contaminated by a substantial threat to internal validity: History. ${ }^{17}$

\section{Training}

After obtaining consent, project researchers conducted two training sessions with the pharmacists and technicians. One session occurred prior to Senior Section implementation - it was designed to describe the project goals and activities and to train pharmacy staff on completing the data collection form (see OTC Encounter Form section below). The second training session, held after pre-intervention data collection, introduced the pharmacy staff to the Senior Section features along with each feature's rationale. Participants received $\$ 30$ for completing the first training and $\$ 60$ for the second training. Each pharmacist and pharmacy technician received this same training, and were trained together. Pharmacists received an additional \$30 for attending a 30-minute training about the most serious adverse reactions relevant to older adults' use of OTC medications. Training for this content was provided by a pharmacist and its content was based on OTC expert guidance and on pharmacy course curricula.

\section{OTC Encounters (OTC Encounter Form)}

An OTC Encounter Form (OTC Form) was developed to capture the characteristics of an encounter between a patient and pharmacy staff. ${ }^{18}$ The OTC Form (included as a Supplementary Appendix) is a 10-item tool consisting of fixed-choice response options representing eight content domains relevant to this study, including who initiated each encounter, activities involved, topics and problems/symptoms discussed, and time spent with the patient. Each pharmacist and/or pharmacy technician involved in a patient encounter completed the OTC Form for that encounter, occurring over a week prior to implementing the Senior Section to allow pharmacy staff to become accustomed to this new activity. The same pharmacists/technicians then used the OTC Form to collect information about patient encounters occurring within two weeks after Senior Section implementation. Pharmacy staff members were compensated $\$ 20$ each week for completing the OTC Forms. One OTC Form was completed per patient encounter, and incentive payment amounts did not depend on the number of completed Forms.

\section{Pharmacy Staff Interviews}

Pharmacists and technicians also completed a 45- to 60-minute semi-structured interview within two months after all the OTC Forms were completed, at the study's conclusion at the end of 2018. The purpose of these interviews was to elicit descriptions of each respondent's role in both the pharmacy and the Senior Section, and how the Senior Section was implemented in their store. Proctor and colleague's taxonomy of implementation outcomes $^{19}$ informed the Interview Guide questions, which provided the data collection structure for each interview. Interviews were conducted (by KZX and another researcher) at a time and location that was convenient for each participant. Pharmacy staff received $\$ 50$ for the interview. All interviews were audio-recorded and transcribed into a de-identified format.

\section{Qualitative Analysis of Interview Data}

A deductive and iterative content analysis approach, guided by the Proctor et al. ${ }^{19}$ taxonomy, was used to identify and explore conceptual categories in the interview data.

Pilot coding. Two researchers (AMG and KZX), using NVivo v.12, independently coded a single pharmacist interview transcript to begin developing coding for all pharmacy staff interviews. The researchers used any coding terms that seemed to apply to relevant interview content. The researchers then met to compare their coding results, focusing on the conceptual or thematic consistency of terms used, whether identified concepts were coded similarly, and whether relevant interview content was overlooked. Discussions were held to determine the final list of codes to be used as the basis for coding remaining interviews. A third researcher (AR) was present during these discussions to adjudicate the deliberation around each code and to help validate the final list of codes.

Final coding. Three weeks after completing the pilot coding, two researchers (AMG and KZX) again independently coded the same pharmacist interview using the finalized coding scheme, then met to review their coding results. This second coding phase identified consistent thematic domains and further validated the final coding scheme. A week later, the same two researchers had independently coded interview transcripts from the second pharmacist and the pharmacy technicians. Coding application was consistent between researchers, demonstrating overall consensus and confirming that the general coding scheme was equally applicable to both pharmacists and pharmacy technicians.

\section{Quantitative Analyses of OTC Form Data}

To compare data from the pre- and post-intervention OTC Forms, frequency distributions were computed for each OTC Form item at both points in time. Items with only one response option, whether dichotomous or multi-categorical, were analyzed as a single variable. Alternatively, for items involving more than one possible response, the response categories were analyzed individually. IBM SPSS Statistics v25ㄷ was used to 
create new variable configurations, and to calculate the frequency distributions and chi-square $\left(\chi^{2}\right)$ tests used for pre/post comparisons of each variable. $P$-values equal to or lower than 0.05 were considered significant.

\section{Mixed-Methods Analysis}

Integrative mixed-methods analysis was used to simultaneously evaluate the findings from the two separate samples - the sample of patient encounter data and the sample of pharmacy staff interview data. ${ }^{20}$ A statistics-by-themes joint display was adapted to present the data, with the statistical results preceding the relevant interview narrative.

\section{Results}

Forty-six OTC Forms were completed throughout the study: 19 at pre-intervention and 27 at post-intervention. Table 1 contains the response frequencies for each relevant OTC Form item, in the order presented on the Form, while Table 2 outlines the responses for the item addressing the numerous potential problems/symptoms involved in a pharmacy staff/patient encounter. Although $\chi^{2}$ analyses were conducted for each OTC Form item, the limited sample size yielded only four viable significant results, which are reported below when related to the discussed theme. Information obtained through pharmacy staff interviews is presented to provide qualitative support for relevant quantitative findings whenever possible, as well as separately according to important thematic domains.

\section{OTC Form Analyses}

Older adults were involved in $53 \%$ of pre-intervention encounters. Despite the Senior Section being designed for older adults, post-intervention older adult encounters dropped slightly to $48.1 \%$, while younger adults (age 18-64) comprised $47.4 \%$ and $48.1 \%$ of patients seen at pre- and post-intervention, respectively. Pharmacy staff also suggested that the Senior Section was attracting teenagers and parents of young children:

.... did see...younger people, high school age even, coming in, picking up some Tylenol, and going to check out. So...everyone of all ages was using it...it wasn't just the older adults. I think people were like, they come in the pharmacy, and they expect medication to be there. It was there. So...all ages were using it, from what I saw. (Pharmacist 1)

...the people that would look for children's medications would ask some questions. And they would see that medication there, and they would [say] "I have a child this age who needs something for a cough." (Pharmacy Technician 1)

Encounters were initiated primarily by patients or their caregivers during both pre- and post-intervention $(100 \%$ and $88.9 \%$, respectively):

[the patient] would definitely just come and ask the pharmacist for assistance. And they would tell the patient to, you know, they'll grab the pharmacist.
And [the pharmacy technicians] would hand it off to the pharmacist. And, you know, myself or the other pharmacist on duty would go over and, you know, kind of get the story of what they were looking for. (Pharmacist 2)

[The Senior Section] prompted people to ask more questions... if it was out in the main store, I don't think they would have asked us...I think the way our pharmacy is set up, I think it prompted more questions and more direct answers, getting the right answers, because our...OTCs were out in the main store, where these [OTCS in the Senior Section are]...where, as I said, the people would feel more comfortable asking questions in the pharmacy. (Pharmacy Technician 1)

However, the rate of encounters initiated by pharmacists, pharmacy technicians or cashiers, or main store employees increased at post-intervention $\left(\chi^{2}(3)=14.087, p=0.003\right)$ :

And so if I did see somebody over there [the Senior Section], or even glancing at it, I would ask them if they needed any help with it. So as I got more comfortable with what was over there, and seeing people, you know, acknowledge it. (Pharmacist 2)

[Once the Senior Section was in place]...I walk up to them and ask if there's anything that I can help, and they explain what they need. And if they have [questions], I can show them the section that we have for the seniors. And if they have further questions concerning if it involves other medication that they're taking, I refer them to the pharmacist. (Pharmacy Technician 1)

Pharmacists provided patient assistance in $52.6 \%$ of all preintervention encounters, but their involvement rose to $63.0 \%$ after Senior Section implementation. Similarly, other pharmacy staff such as technicians demonstrated a $14 \%$ increase in patient interactions ( $68.4 \%$ to $77.8 \%$ ) over the study:

[The Senior Section] just made us a little more willing to maybe go out into the store... (Pharmacist 1)

...my desk was right near the section...so I would sort of call out, ask if they needed some help. Or if they looked confused, you know, just prompt and ask if they need some help, what they're looking for...And then if they had trouble finding it, we would come out and help...I do feel like we were able to help more people just because we could see that they were confused as opposed to, you know, them being out in the aisles and wandering around and not knowing what to look for... (Pharmacy Technician 2) 
Even given increased pharmacy staff involvement in encounters, the need to leave the prescription department for extended periods dropped between pre- and post-intervention (63.2\% to $51.9 \%$, respectively):

,,,since [the Senior Section] was kind of right there in front of the data entry window, sometimes you could just quickly like almost lean over and point to where it was....if they started asking more than one question, that would be, you know, a stimulus to go out around the counter. (Pharmacist 2)

[About being apt to leave the pharmacy box]...I mean, typically, since it was kind of right there in front of the data entry window, sometimes you could just quickly like almost lean over and point to where it was. (Pharmacy Technician 2)

The frequency of prolonged encounters (i.e., encounters lasting 5 to 10 minutes) also decreased from $15.8 \%$ to $3.7 \%$ at postintervention:

...if [patients] needed to find something, it was a lot quicker for us to help them just because it was right there, just a couple steps out. I'd be like, here it is. You know, this is what you need. And it was like, okay, great. So I mean, it took us less time to help them because we didn't have to walk all the way out in the store. (Pharmacist 1)

I feel like it increased the [frequency of encounters], but I also feel like it shortened the length. That's how it felt to me anyway. That we were talking to people more, but we weren't talking to them as long... (Pharmacy Technician 2)

Taken together, these positive changes signify that, although pharmacy staff provided substantial attention to patients during OTC interactions, the Senior Section prompted more staff assistance and perhaps more efficient assistance.

At both pre- and post-intervention, the most prevalent topic discussed during encounters concerned questions about product location ( 89.5 and $74.4 \%$, respectively), but this did show a $17 \%$ post-intervention reduction. The percent increase at post-intervention for other topics discussed are: pharmacy staff product recommendation (64\%), providing specific OTC product details $(111 \%)$, considering self-care appropriateness of OTC use (179\%). The number of topics discussed during each encounter changed after Senior Section implementation, with $57.9 \%$ of encounters addressing one topic at pre-intervention but over half (51.9\%) of encounters representing two or more topics at post-intervention.

Following the implementation of the Senior Section, there were increases in patient interactions relating to problems/symptoms involving OTC medication classes contained in the Senior Section (i.e., medications for allergy, cough/cold, pain, and sleep) $\left(\chi^{2}(1)=11.015, p=0.001\right)$, with concomitant decreases for most other medications (see Table 2). Two specific problems/symptoms also were discussed more often at post-intervention: allergy $\left(\chi^{2}(1)=4.856, p=0.028\right)$ and cough/cold $\left(\chi^{2}(1)=5.810, p=0.016\right) ;$ again medications to treat these symptoms were stocked in the Senior Section. Overall, pharmacy staff believed that the Senior Section contained the OTC products appropriate for the problems/symptoms often discussed during patient encounters:

I think it makes it easier for them just because...it's a more limited section, so they're not overwhelmed by like aisles and aisles of medication. So I think that was a big thing...we had it narrowed down for them. So I think that helps them make a decision better because, you know, a lot of them don't really know where to start. So it's kind of giving them a starting point...here's some Tylenol for pain. Here's something for cough. Here's something for cold. So I think that really helped. (Pharmacist 1 )

I thought [the sleep, pain, and cough, cold, and allergy sections] were great. I thought if it was in the senior or anybody else that came in the pharmacy had any questions, I think seeing that section prompted the people to ask more questions if they had them. (Pharmacy Technician 1)

A final series of items from the OTC Form related to pharmacy staff activities (not just topics discussed) during an OTC encounter. Showing product location to patients was the most prevalent activity at both pre- and post-intervention, beginning at $63.2 \%$ but rising to $74.1 \%$ (likely reflecting the proximity of OTC medication stock within the Senior Section):

Most of the time, yeah, [the relevant medications] were there. Yeah, I didn't have to go out into the store to find them. (Pharmacist 1 )

[Before the Senior Section]...I noticed too a lot of people came in [for a particular medication] and then they would think that they should be in that sort of same section with, you know, the common stuff. And they're, they were in a completely different place where we'd have to help them find it every time. (Pharmacy Technician 2)

There was also a $41 \%$ increase in pharmacy staff providing specific OTC medication recommendations (from $42.1 \%$ to 59.3\%). The remaining measured activities decreased during the study: facilitating non-drug recommendations (77\%), referring to practitioners (30\%), and engaging in other activities (77\%). In fact, of these three activities, the drop in the frequency of "other" pharmacy staff activities achieved significance $\left(\chi^{2}(1)=4.535, p=0.033\right)$. 


\section{Interview Findings}

In addition to the information obtained from the brief OTC Form, interviews of pharmacy staff provide further insights into domains the Form did not directly measure. The interview extractions presented below provide additional support for the benefits of the Senior Section for patient care and medication safety beyond those captured by the Form.

Medication Safety. The Senior Section was conceptualized, created, and implemented principally to enhance patients' safe use of OTC medications. It was apparent throughout the pharmacy staff interviews that the intention of this intervention was unequivocally realized:

I think [the Senior Section contributed to patients choosing medications that were more safe for them to take]. I mean, just because they...had...their choices narrowed down...this is what you have to choose from...this is what is appropriate for you...so let's pick something that's in this category right here. [The patients] weren't like overwhelmed by all these options, all these different combinations of drugs and things. So I think it made it a lot easier. And then they weren't really...worried about like what was in it and.... what other things could be wrong...because there's so many different combinations for the common cold. [I] think the cough and cold thing was huge because this really narrowed it down because, you know, there's Dayquil, NyQuil. There's so many different things, so many different medications, so this was really helpful for people, I believe. (Pharmacist 1)

...it was nice to be able to go and just pick out of these [selected medications]. These are all safe. You don't have to worry. We don't have to worry as much about making sure all of your meds won't interfere or something like that. (Pharmacy Technician 2)

Convenience. Pharmacy staff consistently reported that the Senior Section offered direct accessibility to identify the need for, and initiating, patient engagement:

And so if I did see somebody over there, or even glancing at it, I would ask them if they needed any help with it. So as I got more comfortable with what was over there, and seeing people, you know, acknowledge it...just helping them find specific medications that they were comfortable with. And then also they could browse through it, and kind of get an idea of what they would generally be okay using...And then, you know, they maybe could grab something off the shelf, and quickly look over to the pharmacy and say, "is this okay for something for me to take?" (Pharmacist 2)

Well I, there was times where it was a little easier because you could go, well, it's all over there...It made it easier, because the interruptions, I didn't have to get up and go out to help them find something. I could keep working on what I was working, that multitasking thing. Or if I was on, I would go and I would be on hold a lot because I did, because of the billing thing, so I was able to help people when I was on hold, which was really nice. (Pharmacy Technician 2)

Quality of Pharmacy Staff Consultation. Interviewees described the improved effectiveness of patient encounters subsequent to implementing the Senior Section:

[After the Senior Section was put in place]...[patients] were kind of just like pointing at, oh, what's this? And they just didn't really, they weren't that concerned, they just kind of wondered what the stuff is about...And then we would just quickly explain to them, you know, medication specific for seniors, generally safe for seniors. (Pharmacist 2)

[The Senior Section has made it so that]...we could interact better with them, and they would feel more comfortable...it was more of an enclosed environment, where other people wouldn't be overhearing the questions that they're asking. And they felt more comfortable asking them questions on that medication and even telling the pharmacist what kind of medications they're on. [The patients gained an]...understanding [of] a lot of things, what they could take. (Pharmacy Technician 1)

Efficiency of the Pharmacy Staff Consultation. Pharmacy staff believed that the Senior Section increased the opportunity to make OTC recommendations without having to spend a considerable amount of time guiding patients to products in more distant store locations:

So they would, you know, go to whatever section they would need to, and then they may pick a couple products or that sort of thing and then come to the counter and kind of ask a question. What would you recommend?...Some of them were just asking where things were. And I was like, we actually have it right out here now...more people were probably asking questions than before just because we were right there, and the medication was right there. But you know, similar to the questions we got before when everything was out in the store, but just more so, just more frequent, I guess, just because everything was right there. (Pharmacist 1 )

...we had more questions, but they didn't take us long. There was a lot more just kind of calling out to us behind the counter as opposed to coming up and, you know, waiting in line and asking a question. Just sort of like, hey, where's the thing? And we could go, oh, it's right there. (Pharmacy Technician 2) 
Proximity to the Pharmacy Department. Interview feedback consistently pointed out the advantage of placing the Senior Section close to the prescription department:

So I thought it was a good idea to have some of the main [medications] people look for actually in the pharmacy. And you know, we could just take two steps out, and there we were...I mean, it's more accessible than having to walk from way out in the store. So I did think people felt more, you know, more apt to ask us with the medication being right there. (Pharmacist 1)

I mean, [responding to a patient's needs] would take longer if I had to go out in the main store. We would be away from something else, whereas, the senior section was right there...I think it was better because it's closer. (Pharmacy Technician 1)

Workflow Integration. The Senior Section was designed to minimize its interference with the traditional workflow of pharmacy practice, a goal that was realized:

Oh, you know, it was such a normal thing, before and after, to have an OTC question, that I don't feel like [switching between different tasks] really was anything that I had noticed. (Pharmacist 2)

I think [the workflow] was fine. I just was, everybody was task oriented with different tasks. They could do multiple things at the same time...[the Senior Section did not change things much] except for helping people more but not on our work flow. (Pharmacy Technician 1)

Barriers to the Use of the Senior Section. Importantly, no notable barriers were identified as reducing Senior Section utilization beyond regular work interruptions:

I never got any [complaints]...it didn't really have any sort of impact. It was just, yep, I just, I mean, things kept moving like normal. So it wasn't anything, nothing drastic changed or anything. (Pharmacist 1)

To me, [the patients] seemed like they understood it, and they thought it was a good idea so they didn't have to go far, like I said, to go out... I didn't see any [things that could be barriers]. Everybody thought it was a good idea. (Pharmacy Technician 1)

Notable Observations. Pharmacy staff also provided unprompted feedback that directly addressed the overall value they placed on the Senior Section - for the purpose of this category, a quote is provided from each interviewee:

I mean, like I thought it was a great idea. So I hope you guys can do it in another pharmacy somewhere else, you know, and try it out because I do think it's a good thing to have, and it makes it a lot easier for people to take medications and ask questions... (Pharmacist 1)

I love the idea. (Pharmacist 2)

Everybody thought it was a good idea. (Pharmacy Technician 1)

...we wanted to help people. We were always real big on service and knowing people's names and remembering, you know, what they need and when they need it and helping people personally. So I think the senior section added to that because it made our lives easier. It made it easier to provide that sort of one-on-one service. (Pharmacy Technician 2)

\section{Discussion}

Findings from both the OTC Forms and the pharmacy staff interviews supported the initial benefits of using the Senior Section. Not only did implementing the Senior Section largely maintain pharmacy staff encounters with the target population of older adults, but its impact also extended beyond older adult patients. In fact, people younger than 65 comprised almost half of all encounters, suggesting that the Senior Section has advantages for all patients because of its proximity to pharmacy staff and its medication inventory. Implementing a Senior Section, therefore, could have benefits across age groups, despite the fact that those benefits may not relate to OTC medication safety for older adults.

Although patients initiated a majority of OTC encounters, and did so throughout the project, pharmacy staff initiation of these encounters increased after the Senior Section. In fact, at postintervention pharmacists were involved in providing assistance in almost two-thirds of post-intervention encounters, while pharmacy technicians played a role to an even greater degree. These trends reinforce pharmacy staff as an oft-used patient resource for OTC medication selection. Establishing a Senior Section augmented pharmacy staffs' opportunities to ensure the benefits and lessen the risks of OTC use by assisting older adults in making safe medication decisions. Engagement between pharmacy staff and patients was accomplished without an apparent burden on pharmacy staff workload. Overall, there were fewer prolonged OTC encounters and a greater number of encounters not requiring the pharmacy staff to leave the pharmacy department. Pharmacy staff feedback confirmed that activities prompted by the Senior Section fit into existing workflow, an accomplishment that was a foundational consideration for the SEIPS-inspired intervention.

The proportion of both topics discussed and pharmacy staff tasks during encounters increased in the post-intervention period, while medication recommendations (e.g., providing product details and self-care appropriateness) also became more frequent, suggesting a growing occurrence of clinically 
relevant discussions. Accompanying patients to a product's location also occurred to an even greater extent after Senior Section implementation, while questions about product location diminished slightly (likely due to medication proximity and visibility). Moreover, concomitant reductions in non-drug recommendations, prescriber referrals to discuss clinical issues, and other (unspecified) topics could signify more targeted and goal-driven pharmacy staff/patient interactions - but more work needs to better characterize the precise interaction topics as a means to determine whether these changes are indeed constructive. In addition, the most prominent finding relates to the problems/symptoms discussed at post-intervention. As a single category, problems/symptoms associated with high-risk OTC categories (i.e., pain, sleep, cough/cold, and allergy) demonstrated the most significant increase after Senior Section implementation. Alternatively, problems/symptoms outside the focus of the Senior Section (e.g., eye/ear and first aid) were less likely to be covered during post-intervention encounters. These concomitant changes directly reflect the primary motivation for conceptualizing the Senior Section, and represents the greatest potential for the intervention to effectively reduce OTC misuse and harms. It should be noted, however, that seasonality could have amplified these results, since Senior Section installation occurred in mid-Fall when allergies or cough/cold symptoms are more prevalent; results also may have been different if the study had been conducted during the summer months, when issues related to eye/ear or first aid are ubiquitous.

Taken together, the outcomes from this study support the Senior Section's utility as an effective OTC medication safety intervention within a single pharmacy setting. More frequent encounters - while also being shorter in duration, covering a greater number of topics per encounter, and focusing on issues related directly to the Senior Section medications - all point to increased efficiency of OTC encounters facilitated through the Senior Section implementation. Again, enhanced efficiency in pharmacy staff/patient encounters was an anticipated objective when formulating the intervention design. Further research and adopting the Senior Section in more pharmacies of various sizes and layouts, in collaboration with system engineers, architects, and workflow enhancement technicians, will establish a needed evidence base supporting broader implementation and an understanding of suitable size-to-space ratios.

\section{Limitations}

Although the mixed-methods approach used in this study generated findings that seemed to support clear conclusions, some limitations must still be considered when interpreting the results. First, the number of completed OTC Forms was limited and was largely insufficient to derive statistically significant estimates of change throughout the study. However, pre/post frequencies and percent change calculations suggested effect patterns characterizing the Senior Section's many benefits. Second, qualitative findings were derived from interviews conducted with two pharmacists and two pharmacy technicians. This is a small sample, but the feedback obtained is validated by the fact that it is provided by those pharmacy staff who participated in the study training and completed data collection, and their interview transcripts contained a wealth of relevant information that also substantiated the data gathered from the OTC Forms. Third, these findings relate to the Senior Section implementation in only one pharmacy. Efforts currently are underway (as part of a more comprehensive project) to determine the effect of the Senior Section in other pharmacies, as well as to eventually expand this intervention to a new organization. Fourth, as its name suggests, the Senior Section was designed to address the needs of those within a delimited age range - to improve safe OTC medication use for people 65 and older - because the potential for harms is particularly prevalent for this population due to various

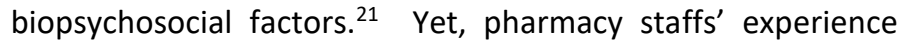
during the study timeframe pointed to its use by other age groups, particularly parents of young children. Since children are at risk for medication-related harms, ${ }^{22}$ further research is needed to investigate the desirability and feasibility of redesigning the intervention to mitigate OTC medication risk for the pediatric population. Fifth, post-intervention data collection occurred over a seven-day period, two weeks after completing implementation. Extended data collection after implementation, as well as at periodic follow-up intervals, would determine the longevity of effects on patient interactions as the Senior Section becomes normalized in practice. Finally, there was no systematic effort to estimate the cost of the intervention, regardless of the variety of benefits observed and measured over the study. Additional efforts are needed to itemize the intervention cost to determine scalability.

\section{Implications}

This study assessed the impact of a physical system redesign on the type, quantity, and duration of patient-pharmacist OTC encounters, by comparing pre-intervention and postintervention encounter results between patients and pharmacy staff and conducting interviews with the pharmacy staff practicing in the pharmacy. The intervention, a speciallydesigned OTC section comprising a stock of OTC medications selected for their lower risk potential to older adults and placed closer to the pharmacy, was conceptualized as an easilyimplemented method to increase the ability of pharmacists to interact with patients about the safe selection and use of OTC medications. Examining this previously untested intervention suggests that a carefully implemented redesign of a community pharmacy has the potential to increase quality and effectiveness of engagements between pharmacy staff and patients without significantly impeding practice workflow, which may in turn substantially reduce OTC-related harms among older adults. Indeed, these findings met the objectives underlying the human factors framework used to develop the Senior Section intervention. Further research is needed to evaluate the generalizability and sustainability of changes that 
occur after intervention implementation, as well as to address patient-centeredness and patient satisfaction metrics according to the Proctor et al. taxonomy of implementation outcomes. ${ }^{18}$ The nascent outcomes derived from this pilot study are encouraging and indicate that the Senior Section could be a valuable tool to improve safe use of OTC medications, not only for older adults but also for all age groups.

Acknowledgements: The authors wish to thank project CoInvestigators Richard Holden PhD, Roger Brown PhD, and Steve Albert PhD, as well as pharmacists Martha Girdaukas and Amy Schellpfeffer, for their assistance in the development the OTC Encounter Form. They also would like to thank the pharmacy staff for completing the OTC Encounter Forms.

Study data were collected and managed using REDCap electronic data capture tools hosted at the University of Wisconsin-Madison, School of Medicine and Public Health (see Paul A. Harris, Robert Taylor, Robert Thielke, Jonathon Payne, Nathaniel Gonzalez, Jose G. Conde, Research electronic data capture (REDCap) - A metadata-driven methodology and workflow process for providing translational research informatics support, J Biomed Inform. 2009 Apr;42(2):377-81, for example). REDCap (Research Electronic Data Capture) is a secure, web-based application designed to support data capture for research studies, providing: 1) an intuitive interface for validated data entry; 2) audit trails for tracking data manipulation and export procedures; 3) automated export procedures for seamless data downloads to common statistical packages; and 4) procedures for importing data from external sources.

\section{Conflict of Interest: None}

Funding Support: This work was supported by the Agency for Healthcare Research and Quality [grant number R18HS024490]; and the Clinical and Translational Science Award (CTSA) program, through the NIH National Center for Advancing Translational Sciences (NCATS) [grant UL1TR000427 (now UL1TR002373)]. The content is solely the responsibility of the authors and does not necessarily represent the official views of either the Agency for Healthcare Research and Quality or the NIH.

\section{References}

1. Colby SL, Ortman JM. Projections of the size and composition of the U.S. population: 2014 to 2060, Current Population Reports (P25-1143). Washington, DC: U.S. Census Bureau; 2014.

2. Krupka LR, Vener AM. Hazards of drug use among the elderly. The Gerontologist. 1979;19(1):90-95.

3. Qato DM, Wilder J, Schumm P, Gillet V, Alexander GC. Changes in prescription and over-the-counter medication and dietary supplement use among older adults in the United States, 2005 vs 2011. JAMA Internal Medicine. 2016;174(4):473-482.

4. Beers MH, Ouslander JG, Rollinher I, Reuben DB, Brooks J, Beck JC. Explicit criteria for determining inappropriate medication use in nursing home residents. Archives of Internal Medicine. 1991;151:1825-1832.

5. The 2019 American Geriatrics Society Beers Criteria Update Expert Panel. American Geriatrics Society 2019 updated AGS Beers Criteria for potentially inappropriate medication use in older adults. Journal of the American Geriatrics Society. 2019. doi: 10.1111/jgs.15767.

6. Budnitz DS, Lovegrove MC, Shehab N, Richards CL. Emergency hospitalizations for adverse drug events in older Americans. New England Journal of Medicine. 2011;356:2002-2012.

7. Albert SM, Roth T, Toscani M, Vitiello MV, Zee P. Sleep health and appropriate use of OTC sleep aids in older adults: Recommendations of a Gerontological Society of America workgroup. The Gerontologist. 2017;57(2):163-170.

8. Chui MA, Stone JA, Martin BA, Croes KD, Thorpe JM. Safeguarding older adults from inappropriate overthe-counter medications: The role of community pharmacists. The Gerontologist. 2014;54(6):989-1000.

9. Sansgiry SS, Bhansali AH, Bapat SS, Xu Q. Abuse of over-the-counter medicines: A pharmacist's perspective. Integrated Pharmacy Research and Practice. 2017;6:1-6.

10. Harris Interactive. Attitudes and beliefs about the use of over-the-counter medicines - $A$ dose of reality: $A$ national survey of consumers and health professionals. Prepared for: National Council on Patient Information and Education; January 2002.

11. Seubert L, Whitelaw K, Hattingh L, Watson MC, Clifford RM. Development of a theory-based intervention to enhance information exchange during over-the-counter consultations in community pharmacy. Pharmacy. 2018;6, 117; doi:10.3390/pharmacy6040117. 
12. American Pharmacists Association. Practice Insights: Pharmacists as Self-Care Advisors. Washington, DC: American Pharmacists Association; December 2015.

13. Chui MA, Stone JA, Holden RJ. Improving over-thecounter medication safety for older adults: A study protocol for a demonstration and dissemination study. Research in Social and Administrative Pharmacy. 2017;13:930-937.

14. Reddy A, Lester CA, Stone JA, Holden RJ, Phelan CH, Chui MA. Applying participatory design to a pharmacy system intervention. Research in Social and Administrative Pharmacy. 2018 Nov 27. pii: S15517411(17)30866-5. doi: 10.1016/j.sapharm.2018.11.012. [Epub ahead of print].

15. Nastasi BK, Hitchcock J, Sarkar S, Burkholder G, Varjas $\mathrm{K}$, Jayasena A. Mixed methods in intervention research: Theory to adaptation. Journal of Mixed Methods Research. 2007;1(2):164-182.

16. Holden RJ, Carayon P, Gurses AP, Hoonakker P, Hundt AS, Ozok AA, Rivera-Rodriguez J. SEIPS 2.0: a human factors framework for studying and improving the work of healthcare professionals and patients. Ergonomics. 2014;56(11):1669-1686.

17. Slack MK, Draugalis JR. Establishing the internal and external validity of experimental studies. American Journal of Health Systems Pharmacies. 2001;58(22):2173-2181.
18. Gilson AM, Stone JA, Reddy A, Chui MA. Exploring how pharmacists engage with patients about overthe-counter medications. Journal of the American Pharmacists Association. 2019 Sep 6. pii: S15443191(19)30394-2. doi: 10.1016/j.japh.2019.08.001. [Epub ahead of print].

19. Proctor $E$, Silmere $H$, Raghavan $R$, Hovmand $P$, Aarons G, Bunger A, Griffey R, Hensley M. Outcomes for implementation research: conceptual distinctions, measurement challenges, and research agenda. Administration and Policy in Mental Health and Mental Health Services Research. 2011;38(2):65-76.

20. Guetterman TC, Fetters MD, Creswell JW. Integrating quantitative and qualitative results in health science mixed methods research through joint displays. Annals of Family Medicine. 2015;13(6):554-561.

21. Paliwal Y, Gendron TL, Jones RM, Moczygemba L, Nadpara PA, Slattum PW. A qualitative study to understand over-the-counter medication use and decision-making among residents of senior-living communities. Research in Social and Administrative Pharmacy. 2018;15(6):730-737.

22. Rieder M. Adverse drug reactions in children: Pediatric pharmacy and drug safety. The Journal of Pediatric Pharmacology and Therapeutics. 2019;24(1):4-9. 
Table1. Frequency of OTC Form Items at Pre- and Post-Intervention (the Senior Section)

\begin{tabular}{|c|c|c|}
\hline OTC Form Items & $\begin{array}{l}\text { Cases at Pre- } \\
\text { Intervention } \\
\text { (Frequency \%) }\end{array}$ & $\begin{array}{l}\text { Cases at Post- } \\
\text { Intervention } \\
\text { (Frequency \%) }\end{array}$ \\
\hline \multicolumn{3}{|l|}{ Age of patient involved in encounter } \\
\hline Older adult (ages $\geq 65$ ) & $10(52.6)$ & $13(48.1)$ \\
\hline Adult (ages 18-64) & $9(47.4)$ & $13(48.1)$ \\
\hline Child/Adolescent (ages < 18) & $0(0)$ & $1(3.7)$ \\
\hline \multicolumn{3}{|l|}{ Topics discussed during OTC encounter } \\
\hline Product location & $17(89.5)$ & $20(74.1)$ \\
\hline Product recommendations & $6(31.6)$ & $14(51.9)$ \\
\hline Product details & $3(15.8)$ & $9(33.3)$ \\
\hline Self-care appropriateness & $1(5.3)$ & $4(14.8)$ \\
\hline Other & $1(5.3)$ & $0(0)$ \\
\hline Pharmacy staff left the pharmacy box to help patient & $12(63.2)$ & $14(51.9)$ \\
\hline \multicolumn{3}{|l|}{ Initiated patient encounter } \\
\hline Pharmacy staff & $0(0)$ & $3(11.1)^{*}$ \\
\hline Patient or caregiver & $19(100)$ & $24(88.9)$ \\
\hline \multicolumn{3}{|l|}{ Staff that assisted with encounter } \\
\hline Pharmacist only & $6(31.6)$ & $6(22.2)$ \\
\hline Pharmacy technician/cashier only & $9(47.4)$ & $10(37.0)$ \\
\hline Pharmacy student/intern only & $4(21.1)$ & $0(0)$ \\
\hline Combination of staff & $0(0)$ & $11(40.7)$ \\
\hline \multicolumn{3}{|l|}{ Staff activity during encounter } \\
\hline Showed patient location of product & $12(63.2)$ & $20(74.1)$ \\
\hline Recommended OTC medication & $8(42.1)$ & $16(59.3)$ \\
\hline Non-drug recommendation & $3(15.8)$ & $1(3.7)$ \\
\hline Referred to physician or other healthcare practitioner & $2(10.8)$ & $2(7.4)$ \\
\hline Other & $6(31.6)$ & $2(7.4)^{*}$ \\
\hline \multicolumn{3}{|l|}{ Time staff spent with patient regarding OTC question } \\
\hline Less than 1 minute & $8(42.1)$ & $10(37.0)$ \\
\hline 1-3 minutes & $7(36.8)$ & $13(48.1)$ \\
\hline 3-10 minutes & $4(21.1)$ & $4(14.8)$ \\
\hline
\end{tabular}

${ }^{*} \chi 2$ values were significant 
Table 2. Types of problems/symptoms involved in OTC encounters

\begin{tabular}{|l|c|c|c|}
\hline Problem/Symptom & $\begin{array}{c}\text { Cases at Pre- } \\
\text { Intervention } \\
\text { (Frequency \%) }\end{array}$ & $\begin{array}{c}\text { Cases at Post- } \\
\text { Intervention } \\
\text { (Frequency \%) }\end{array}$ & Percent Change \\
\hline Allergy* & $0(0)$ & $6(22.2)$ & -- \\
\hline Cough/Cold* & $0(0)$ & $7(25.9)$ & -- \\
\hline Eye or Ear & $3(15.8)$ & $1(3.7)$ & $-77 \%$ \\
\hline First Aid & $7(36.8)$ & $5(18.5)$ & $-50 \%$ \\
\hline Gastrointestinal & $0(0)$ & $1(3.7)$ & -- \\
\hline Heart Health & $0(0)$ & $2(7.4)$ & -- \\
\hline Pain* & $1(5.3)$ & $4(14.8)$ & $+179 \%$ \\
\hline Sleep Trouble* & $0(0)$ & $0(0)$ & $0 \%$ \\
\hline Vitamin/Supplement Question & $1(5.3)$ & $3(11.1)$ & $+109 \%$ \\
\hline Other & $8(42.1)$ & $3(11.1)$ & $-74 \%$ \\
\hline Combinations of problems/symptoms & $1(5.3)$ & $5(18.5)$ & $+249 \%$ \\
\hline
\end{tabular}

* Problems/symptoms related to the OTC medications comprising the Senior Section inventory, which were associated with significant $\chi 2$ values 